\title{
LE RÔLE DE L'AFFIRMATION DE L'IDENTITÉ HOMOSEXUELLE PARENTALE DANS L'EXPÉRIENCE DES ENFANTS AYANT UN PÈRE GAI OU UNE MÈRE LESBIENNE
}

\author{
STÉPHANIE LAVOIE, DANIELLE JULIEN et CHRISTIANE FORTIER \\ Université du Québec à Montréal
}

\begin{abstract}
RÉSUMÉ
La présente étude porte sur les expériences autorapportées de 19 jeunes adultes, 11 femmes et 8 hommes, qui ont vécu leur enfance et leur adolescence avec un père gai ou une mère lesbienne né(e) dans les années 1950 ou 1960. Le contenu des entrevues a été classifié et analysé en fonction d'une grille de catégories inspirées du modèle écologique de Bronfenbrenner (1986), et en fonction de l'influence structurante du coming out des parents sur l'expérience des enfants. L'analyse de contenu montre que les enfants d'un père gai ou d'une mère lesbienne qui cache ou qui n'accepte pas son identité homosexuelle vivent plus d'inconfort et ont davantage de réactions négatives que ceux dont le parent affirme son identité homosexuelle. Les résultats montrent aussi que les enfants qui ont un parent qui affirme le fait d'être gai ou lesbien ont des réactions et des relations plus positives avec ce parent comparativement à ceux dont le parent le cache ou ne l'accepte pas. Finalement, les analyses indiquent que la transition de l'enfance à l'adolescence amène des difficultés singulières chez les enfants de parents qui cachent ou n'acceptent pas leur propre identité sexuelle, telle la peur de la réaction des autres, l'homophobie intériorisée et la crainte de devenir homosexuel. Ces difficultés semblent persister à l'age adulte.
\end{abstract}

Depuis la dernière décennie, les groupes pour la défense des droits des minorités sexuelles ainsi que la reconnaissance de la variabilité des structures familiales ont favorisé une plus grande visibilité de l'homoparentalité. La famille homoparentale se caractérise par la présence d'au moins un homme gai ou une femme lesbienne vivant avec un enfant biologique ou adopté. Au Québec, certaines initiatives, telles l'Association des mères lesbiennes de Montréal (AML, depuis 1998) ou l'Association des pères gais de Montréal (APGM, depuis 1990) et l'adoption récente au des lois 32 (1999) et 84 (2002) au Québec, le jugement de la Cour d'appel du Québec (2004) et l'adoption de la loi fédérale C-38 (2005) sur l'union de fait, l'union civile et le mariage entre deux personnes de même sexe ont contribué aux changements des droits, privilèges et obligations des parents au sein des familles homoparentales.

Ces avancées ont été facilitées en partie par l'avancée des connaissances empiriques cumulées au cours des 20 dernières années. Les recherches sur les enfants de parents homosexuels montrent qu'ils ne se distinguent pas des autres enfants quant au développement de leur identité sexuelle. De plus, ils ne sont pas davantage abusés sexuelle-

Cette recherche a été possible grâce à une subvention du Conseil de recherche en sciences humaines du Canada, no. 410-2002-1455. Toute correspondance concernant cet article devrait être acheminée à Stéphanie Lavoie, a/s Danielle Julien, julien.danielle@uqam.ca 


\section{REVUE CANADIENNE DE SANTÉ MENTALE COMMUNAUTAIRE}

ment et se développent de manière comparable aux autres enfants des points de vue affectif, sexuel et cognitif, ainsi que de celui de leur intégration sociale à l'école (pour une revue de la recherche empirique, voir notamment Julien, Dubé, \& Gagnon, 1994; Nadaud, 2002; Patterson, 2000; et pour une méta-analyse des recherches sur les enfants, voir Allen \& Burrell, 1996).

La majorité des recherches menées à la fin des années 80 et au cours des années 90 portent sur la normalité des enfants et des adolescents et adolescentes. Afin de documenter les effets de l'homosexualité des parents sur le développement des enfants, ces études ont comparé les enfants de familles homoparentales à des groupes témoins d'enfants nés et vivant dans des familles hétéroparentales. Cette préoccupation a limité et retardé l'analyse de l'expérience spécifique aux enfants vivant dans une famille en marge des normes hétéroparentales, puisque les dimensions propres à la marginalité et à la stigmatisation, non comparables entre les familles homoparentales et hétéroparentales, n'étaient pas incluses dans l'analyse.

La présente étude a pour objectif de décrire les expériences autorapportées de jeunes adultes qui ont vécu avec un père gai ou une mère lesbienne né(e) dans les années 1950 et 1960. Les données recueillies permettent de documenter, selon les perceptions de jeunes adultes, le contexte de développement de l'enfant à une époque où les attitudes envers l'homosexualité étaient particulièrement stigmatisantes. De plus, comme les premières études portaient en général sur de jeunes enfants ayant une capacité limitée d'analyse de leur situation familiale, les entrevues avec des jeunes adultes permettent non seulement de recueillir des témoignages et des analyses ayant un certain recul, mais aussi de dresser un tableau des représentations de leur passage de l'enfance à l'adolescence dans une famille homoparentale.

Si les enfants nés aujourd'hui de parents gais ou lesbiens peuvent espérer vivre leur enfance et leur adolescence dans un contexte encadré par des politiques sociales de plus en plus inclusives des familles homoparentales, l'expérience des enfants nés à une époque où les minorités sexuelles subissaient l'exclusion et la stigmatisation des institutions et de leurs milieux de vie est peu connue. Dans la plus grande partie du siècle dernier, l'homosexualité a été jugée comme déviante ou pathologique et, par conséquent, incompatible avec la parentalité. Malgré ce climat, plusieurs personnes gaies et lesbiennes ayant eu des enfants dans le cadre d'un mariage ou d'une union hétérosexuelle ont fait le choix de mettre fin à leur relation conjugale tout en souhaitant avoir la garde de leurs enfants. En 1973, l'American Psychiatric Association excluait l'homosexualité de la liste des psychopathologies, et en 1977 au Québec, l'article 10 de la charte des droits et libertés indiquait qu'il devenait discriminatoire de refuser la garde d'un enfant à un parent uniquement en fonction de son orientation sexuelle (Deleury, 1984). Malgré ces avancées des années 70, les familles homoparentales ont vécu avec la présomption que les enfants de ces familles seraient psychologiquement mal adaptés, souffriraient eux-mêmes de stigmatisation et deviendraient homosexuels (e.g., Perez, DeBord, \& Bieschke, 2000).

Le modèle écologique de Bronfenbrenner (1986) guide notre analyse des expériences de vie de ces jeunes adultes. Ce modèle stipule que l'individu (ontosystème) est au centre de systèmes emboîtés les uns dans les autres qui influencent son développement. Dans la présente étude, notre attention porte sur les effets de l'hétérosexisme en tant qu'axe transversal qui module l'ensemble des sous-systèmes du modèle. Les données recueillies réfèrent aux microsystèmes de l'enfant, c'est-à-dire sa vie familiale, sa vie scolaire et sa vie au sein de son réseau de pairs. Elles portent aussi sur le mésosystème de l'enfant, lequel réfère aux interactions entre les différents microsystèmes dans lesquels l'enfant est directe- 


\section{LE RÔLE DE L’AFFIRMATION DE L’IDENTITÉ HOMOSEXUELLE}

ment impliqué. Par exemple, le modèle présume que l'appartenance de l'enfant à une famille stigmatisée et les dynamiques familiales que ce statut engendre modulent les expériences de l'enfant dans son réseau de pairs, lesquelles peuvent en retour affecter son expérience familiale. Notre lecture a pour arrière-plan la place déterminante de l'exosystème de l'époque caractérisé par des politiques sociales et familiales hétérocentrées, et du macrosystème, c'est-à-dire des idéologies, croyances et valeurs sociales stigmatisantes qui ont marqué le développement de l'identité sexuelle des adultes de cette époque.

Inspirées par ce modèle, nous stipulons que dans les sociétés comme la nôtre, marquée par de rapides changements sociaux, chaque nouvelle cohorte d'individus est exposée à un macrosystème caractérisé par des conditions historiques et sociales particulières. Bien que l'homosexualité ait été décriminalisée au Canada en 1969, les parents des jeunes adultes interviewés ont vécu leur jeune vie d'adulte et ont éduqué leurs enfants à une époque où l'homosexualité était tabou, condamnée par le clergé et perçue comme une maladie ou un crime. Ces attitudes sont définies aujourd'hui comme hétérosexistes. L'hétérosexisme réfère à tout système idéologique qui dénie, dénigre et stigmatise toute forme de comportements, d'identités et de relations non hétérosexuels (Herek, 1995). Les années 60 sont aussi marquées par la transformation des structures familiales prédominantes, la famille nucléaire traditionnelle cédant le pas à l'aménagement de nouvelles formes familiales, telles que les familles monoparentales, recomposées et adoptives (e.g., Lapierre-Adamcyk, 2001).

Les enfants nés dans les années 70 et 80 de parents gais et lesbiens sont, au cours de cette période, témoins du plein essor du mouvement de libération des gais et lesbiennes des pays anglo-saxons. Néanmoins, à cette époque, les mères qui se séparent d'un conjoint pour vivre leur lesbianisme prennent la sellette lors de litiges concernant la garde des enfants et perdent la plupart du temps la garde de l'enfant en raison de présumées difficultés que les enfants sont susceptibles de rencontrer du fait d'être éduqués par une mère non hétérosexuelle (e.g., Editors of Havard Law Review, 1990; Green, 1992). Plusieurs d'entre elles préfèrent alors ne pas divulguer leur identité homosexuelle et vivre dans la clandestinité afin d'obtenir la garde de leurs enfants. Quant aux pères gais de cette époque, tout comme les mères lesbiennes, ils voient naître leurs enfants dans le cadre d'unions hétérosexuelles soit parce qu'ils ignorent ou nient leur attirance pour les hommes, soit parce qu'ils désirent avoir des enfants malgré leur attirance pour les personnes de même sexe (Barret \& Robinson, 1990; Bigner \& Bozett, 1989; Bigner \& Jacobsen, 1989; Bozett, 1987).

Outre les très rares données de recherche empirique sur le développement de l'identité homosexuelle à l'âge adulte lors du passage de l'hétérosexualité à l'homosexualité, nous ne connaissons rien des processus de divulgation de l'identité homosexuelle (coming out) des parents gais et lesbiens à leurs enfants, ni des effets du silence ou du coming out sur leurs enfants et les dynamiques familiales. Selon Dunne (1987), les pères gais ex-hétérosexuels participant à des groupes de discussion visant à les soutenir dans la divulgation de leur identité homosexuelle à leurs enfants rapportent que le secret entourant leur identité sexuelle génère de l'anxiété intense. De même, des témoignages recueillis par Bozett (1980, 1981) indiquent que le silence des parents, motivés par la peur de perdre leurs enfants, entraîne en retour une distance psychologique qui nuit au rapprochement affectif avec leurs enfants.

Nous nous attendons à ce que les adolescents et adolescentes des années 80 et 90 qui ont un parent homosexuel aient vécu bien souvent dans un milieu familial caractérisé par le silence (in the closet) et l'ambivalence reliés à l'orientation homosexuelle de leurs parents, 


\section{REVUE CANADIENNE DE SANTÉ MENTALE COMMUNAUTAIRE}

malgré l'émergence d'un certain libéralisme envers l'homosexualité. D’autre part, puisque l'adolescence est une période charnière du développement identitaire qui exacerbe la sensibilité aux jugements des pairs, certains adolescents et adolescentes de cette époque, conscients de l'orientation sexuelle de leur père ou de leur mère, ont probablement été plus à risque d'être touchés par la stigmatisation des pairs à leur égard et à l'égard de leur parent.

À la lumière des aspects historiques et des valeurs sociales susceptibles de marquer le vécu des parents gais et lesbiens et celui de leurs enfants, la présente étude examine les perceptions rétrospectives de jeunes adultes qui ont vécu leur enfance et leur adolescence au cours des années 80 et 90 dans une famille dont le père gai ou la mère lesbienne est né(e) dans les années 50 ou 60 . Tenant compte des théories du développement, elle examine aussi les difficultés rapportées par les jeunes lors de leur passage de l'enfance à l'adolescence. Cette étude se veut exploratoire et ses résultats devraient permettre le développement d'une recherche des dynamiques familiales qui inclurait des dyades parents-enfants appartenant à deux cohortes.

\section{MÉTHODE}

\section{Participants et participantes}

Dix-neuf jeunes adultes en provenance de la région de Montréal-11 femmes (âge moyen $=23$ ans, ÉT $=3.66$ ) et 8 hommes (âge moyen $=20$ ans, ÉT $=3.20$ )—participent à l'étude. Ils sont recrutés en partie lors d'événements publics réunissant des gais et lesbiennes d'âges variés dont certain(e)s sont susceptibles d'avoir des enfants jeunes adultes. Ils sont aussi recrutés au moyen d'annonces dans les journaux invitant de jeunes adultes à témoigner de leur vécu en tant qu'enfant d'un ou de parents gais ou lesbiens. Pour être inclus dans l'échantillon, les jeunes adultes doivent répondre aux critères suivants: (a) être âgé(e) entre 18 et 30 ans, (b) avoir la certitude que l'un de ses parents est gai ou lesbien et (c) avoir vécu au moins une partie de la période de l'adolescence conscient de l'orientation homosexuelle de son parent. L'entrevue avec un participant n'a pas été retenue, car celui-ci ne pouvait certifier que son père était gai. Parmi les hommes, 4 ont une mère lesbienne et 4 ont un père gai. Parmi les femmes, 7 ont une mère lesbienne et 5 ont un père gai. Tous les participants et participantes sont d'origine canadienne (95\%) à l'exception de l'un qui est d'origine européenne. Tous les participants et participantes parlent couramment le français. Quant à la scolarité, 31,5\% ont terminé des études secondaires, 31,5\% des études collégiales ou l'équivalent et $37 \%$ ont terminé un baccalauréat à l'université; $26 \%$ des participants et participantes sont aux études, 15,5\% travaillent à temps plein, $11 \%$ travaillent à temps partiel et les autres combinent travail et études ou ont une autre situation. La difficulté de recruter cette population a limité notre capacité de diversifier la composition de l'échantillon en fonction de variables pertinentes telles l'appartenance à des minorités culturelles, les structures familiales homoparentales, le niveau socio-économique, le moment du coming out du parent, l'attitude du parent hétérosexuel à l'égard de l'homosexualité de l'autre parent, etc. Le potentiel de transférabilité des résultats de cette étude est donc limité.

\section{Procédure}

Lors d'un premier contact téléphonique, les participantes et participants sont informés que l'étude vise à mieux connaître la vie des jeunes qui ont vécu avec un parent homosexuel au cours des années 80-90. Suivant la description de la rencontre, les 


\section{LE RÔLE DE L’AFFIRMATION DE L’IDENTITÉ HOMOSEXUELLE}

participantes et participants sont invités à une rencontre d'entrevue semi-structurée dans les locaux de l'institution universitaire. L'intervieweur possède une formation clinique à l'entrevue et est membre de l'Ordre des psychologues du Québec, et la procédure de rencontre a reçu l'approbation du comité d'éthique de la recherche de l'institution. Lors de leur visite, les participantes et participants signent une feuille de consentement éclairé et remplissent un questionnaire d'informations démographiques. La consigne présentée au participant ou à la participante consiste à décrire son expérience en tant qu'enfant de parent homosexuel. Au cours de l'entrevue, l'intervieweur invite le participant ou la participante à décrire (a) la qualité de ses rapports au parent homosexuel, pendant l'enfance et l'adolescence, en lien avec l'homosexualité du parent, (b) les difficultés vécues à l'intérieur de la famille en rapport avec l'identité sexuelle du parent, (c) les sources perçus de ces difficultés, (d) l'impact perçu de sa marginalité familiale dans ses rapports à l'école et avec son réseau de pairs, (e) l'impact perçu de l'identité sexuelle du parent sur sa propre identité sexuelle, et enfin (f) la qualité de sa relation actuelle avec son parent. Les entrevues durent en moyenne 40 minutes (ÉT $=17.32$ ). Un montant de $20 \$$ est remis aux participants et participantes après la rencontre. Les entrevues sont enregistrées et sont retranscrites pour fin d'analyse.

\section{Méthode d'analyse}

Catégorisation et classification. Deux analystes procèdent d'abord à une première lecture libre de chaque verbatim afin de se familiariser avec son contenu. Étant donné l'importance théorique des transitions de développement (enfance, adolescence, âge adulte) et de celle des systèmes (famille, école, amis et amies) dans lesquels l'enfant se développe, ces variables guident la première étape de la méthode de catégorisation. Le contenu est catégorisé selon un système chronologique composé de trois périodes: enfance (0-11 ans), adolescence (12-17 ans) et âge adulte (18 ans et plus), constituant les balises d'un chronosystème. Pour chacun(e) des participants et participantes, un fichier est créé dans lequel sont insérés les propos qui correspondent à la période chronologique appropriée. Puis, à l'intérieur de chaque période du système chronologique, le contenu est catégorisé selon cinq contextes inspirés du modèle de Bronfenbrenner (1986):

1. L'Ontosystème réfère aux comportements du participant ou de la participante par rapport à lui-même ou elle-même (qui ne fait pas référence directement à l'école, à la famille ou aux amis et amies) ainsi qu'aux perceptions et aux réflexions du participant ou de la participante sur sa propre personne (ex: je suis hétérosexuel, j’ai une excellente santé, etc.).

2. Le microsystème Famille réfère aux propos concernant la perception et l'histoire de vie des membres de la famille du participant ou de la participante, incluant les parents biologiques, les coparents, les frères et sœurs et la famille élargie. Sont inclus dans ce microsystème les propos qui ont trait à la perception des amis et amies, du chum ou de la blonde, du conjoint ou de la conjointe ou de l'époux ou l'épouse des parents biologiques du participant ou de la participante ainsi que leur famille, et les propos qui se rapportent à l'époux ou l'épouse du participant ou de la participante lorsqu'il y a des enfants issus de cette union.

3. Le microsystème Réseau social réfère aux propos concernant spécifiquement la perception de la relation du participant ou de la participante avec ses amis et amies, son ami ou son amie de cœur, son conjoint ou sa conjointe ainsi que les ami(e)s et la famille de chacun(e) de ceux-ci. 


\section{REVUE CANADIENNE DE SANTÉ MENTALE COMMUNAUTAIRE}

4. Le microsystème École se rapporte aux propos abordant le vécu du participant ou de la participante dans les institutions d'enseignement et à la perception de la vie des étudiant(e)s, du corps professoral et de la dynamique entre ces acteurs de ces lieux d'enseignement.

5. Le contexte Maintenant réfère aux propos abordant les perceptions, réflexions et critiques de l'interviewé(e) par rapport à tous ses milieux de vie et ses différents acteurs ainsi que les propos qui se rapportent aux messages que le participant ou la participante aimerait transmettre à la société.

Condensation. Dans un deuxième temps, le travail consiste à donner un sens aux très longs contenus intégrés dans ces cinq sous-systèmes. Pour ce faire, chaque regroupement de phrases qui représentent une idée ou un événement est résumé par l'expression la plus concise et la plus précise afin de créer des mots clés (processus de condensation, Kvale, 1996). Un corpus de définitions (dictionnaire) des mots clés est élaboré afin d'établir des critères décisionnels pour la classification des contenus. Par exemple, le mot clé homophobie réfère à tout énoncé manifestant du dénigrement, de la haine, ou du rejet en raison d'une orientation homosexuelle.

Analyse de contenu proprement dite. Au cours de la première lecture libre des verbatim, les deux analystes ont aussi repéré la récurrence du thème de l'affirmation (ou de la non-affirmation) du parent de sa propre orientation homosexuelle ainsi que ses conséquences structurantes sur l'expérience des participants et participantes. Par définition, l'affirmation réfère à l'expression d'idées et de comportements affichant publiquement l'identité homosexuelle ainsi que la valorisation positive de ces manifestations. Ce repérage au premier niveau de lecture libre des verbatim est guidé par la fonction théorique fondamentale du coming out dans le développement identitaire des personnes homosexuelles et dans la qualité des liens entre les personnes homosexuelles et leur entourage proche (e.g., Savin-Williams \& Esterberg, 2000). L'affirmation par le parent de son homosexualité a donc constitué une catégorie majeure servant à classifier l'ensemble des mots clés. Voici un exemple d'affirmation (A) et un autre de non-affirmation (NA) tirés des entrevues: Elle avait des relations affectueuses avec des femmes quand j'avais 3-4 ans, représentation d'un comportement d'affirmation de la mère, classifié A; Mon père n'a jamais fait de coming out, il n'a jamais avoué ce que mon frère nous avait déjà dit de la sexualité de mon père, représentation du tabou et d'un comportement non affirmatif, classifié NA.

Tous les mots clés et leur contenu original ont été lus et sélectionnés par les deux analystes selon leur pertinence avec le degré d'affirmation par le parent de sa propre homosexualité. Les énoncés référant aux causes et aux effets possiblement reliés à la variable affirmation étaient classifiées de la même façon que les mots clés qui y étaient reliés de façon directe. L'occurrence des deux codes (A et NA) est ensuite dénombrée pour chacune des entrevues, permettant ainsi de classifier chaque participant ou participante en fonction du degré d'affirmation par le parent de sa propre orientation homosexuelle (catégorisation, Kvale, 1996). À la suite de cette procédure, deux groupes sont constitués. Le groupe A comprend les participants et participantes dont l'occurrence de code A est supérieure à celle de NA et le groupe NA comprend ceux dont le nombre de code NA est supérieur à celui de A. Dans la présente recherche, l'échantillon est composé de 7 enfants avec des parents affirmatifs (groupe A) et de 12 participants et participantes avec des parents non affirmatifs (groupe NA).

Création de thèmes. Un troisième niveau de catégorisation consiste à classifier les mots clés de chaque participant ou participante, maintenant jumelés à un code A ou NA, en 


\section{LE RÔLE DE L’AFFIRMATION DE L’IDENTITÉ HOMOSEXUELLE}

fonction de leurs similitudes et de leurs distinctions avec ceux des autres participants et participantes et ce, pour chacune des trois périodes du chronosystème. Pour ce faire, plusieurs mots clés sont regroupés dans une même catégorie réduite à une expression concise donnant ainsi des thèmes permettant l'analyse proprement dite. Par exemple, les rapports négatifs avec les amis et amies pendant l'enfance (thème) sont analysés en fonction des mots clés, jumelés aux codes A et NA, référant à ces expériences négatives des participants et participantes.

Accords inter-juges. Des accords par consensus de classification ont été réalisés avec les contenus de 17 participants et participantes sur 19 pour chacune des étapes de l'analyse des données.

Les résultats qui suivent présentent d'abord les thèmes communs aux deux groupes, puis les thèmes qui les distinguent. Étant donné l'espace limité de cet article, les exemples de thèmes ne seront présentés que dans la section portant sur les différences.

\section{RÉSULTATS}

\section{Thèmes communs partagés par les deux groupes}

Tous les participants et participantes de l'étude (sauf une participante dont les parents se sont séparés avant sa naissance), rapportent avoir vécu à un moment ou un autre le divorce ou la séparation de leurs parents biologiques. Ils ont tous eu également à affronter les réactions du parent hétérosexuel (sauf une participante dont les deux parents séparés ont affiché une orientation homosexuelle après leur séparation). Certains participants et participantes ont vécu exclusivement avec le parent soit hétérosexuel soit homosexuel; d'autres ont vécu dans un contexte de garde partagée. Dans les deux cas, ils ont vécu avec ou sans les nouveaux conjoint(e)s des parents séparés. Une large proportion de participants et participantes des deux groupes rapportent avoir eu des réactions négatives à la transition familiale et avoir eu une relation problématique avec leur père gai ou leur mère lesbienne soit durant l'enfance, durant l'adolescence, à l'âge adulte ou à plus d'une période. Aussi, la grande majorité des participants et participantes rapportent, à différentes périodes de leur vie, des propos reliés à la non-affirmation du parent de sa propre orientation homosexuelle. L'inconscience, le tabou et l'isolement par rapport aux amis et amies et au milieu scolaire sont des thèmes communs et récurrents aux deux groupes (A et NA) durant l'enfance.

À l'adolescence, les participants et participantes se questionnent sur leur propre orientation sexuelle et, pour plusieurs, leur orientation s'affirme clairement. Dans notre échantillon, 50\% des garçons se sont définis d'orientation homosexuelle et $17 \%$ des filles se sont définies d'orientation bisexuelle. Les participants et participantes mentionnent que cette période est difficile car ils vivent des difficultés personnelles au sein de leur famille, auprès de leurs amis et amies et dans le milieu scolaire. Par exemple, pour plusieurs, l'homosexualité est un sujet tabou; certain(e)s ont un cercle d'amis et amies restreint; et d'autres craignent la réaction des autres. Dans les groupes A et NA, certains participants et participantes mentionnent que leur parent gai ou lesbien vivait des difficultés personnelles. Toutefois, si les enfants des deux groupes rapportent avoir vécu des difficultés en lien avec l'homosexualité de leur parent, l'expérience des enfants ayant un parent non affirmatif apparaît plus négative que celle des enfants ayant un parent affirmatif. 


\section{REVUE CANADIENNE DE SANTÉ MENTALE COMMUNAUTAIRE}

\section{Distinctions entre les enfants ayant un parent homosexuel qui affirme son identité (groupe A) et les enfants ayant un parent homosexuel qui ne l'affirme pas (groupe NA)}

L'ensemble des participants et participantes rapportent fort peu de thèmes par rapport à eux-mêmes pour la période de l'enfance. Cependant, davantage de membres du groupe NA comparés à ceux du groupe A mentionnent qu'ils étaient plutôt inconscients de l'orientation homosexuelle de leur parent pendant l'enfance. Aussi plus de membres du groupe NA révèlent avoir remarqué que leur parent passait de relations homosexuelles à hétérosexuelles et vice-versa durant cette période. Durant l'enfance, si davantage de membres du groupe NA comparé au groupe A ont mentionné qu'ils trouvaient normal le fait d'avoir un père gai ou une mère lesbienne, ils sont aussi plus nombreux à mentionner que l'homosexualité de leur parent était un sujet tabou dans la famille:

Chez nous, elles ont toujours eu leur chambre séparée, alors, déjà moi je n’avais pas

d'indice, c'était en tous cas bien caché (fille de mère lesbienne NA).

Les enfants NA avaient une relation plus troublé avec leur parent gai ou lesbien qui cache son homosexualité. Voici l'exemple d'un garçon NA, dont la famille maintient le tabou sur l'homosexualité de la mère, et qui, à l'âge de 10 ans, entend sa mère faire l'amour avec une femme:

J'en n'avais pas dormi de la nuit, ou très peu quoi, je lui en voulais beaucoup, j'avais envie d'entrer dans la chambre et de la taper.

Aussi, les membres du groupe NA sont plus nombreux à mentionner avoir eu des réactions négatives et avoir vécu un certain malaise envers l'homosexualité de leur parent:

C'est sûr que, dans mon enfance, il y avait un grand malaise tout le temps et je pense que ça s'est répercuté sur toute la famille. C’est peut-être très sévère, mais j’ai l'impression que le noyau, c'était quand même l'homosexualité de mon père. S'il l'avait acceptée plus jeune, je ne pense pas que ce malaise-là aurait été présent (garçon de père gai NA).

Les rapports avec les amis et amies durant l'enfance distinguent aussi les enfants des deux groupes. Alors que les enfants $\mathrm{A}$ parlaient du fait d'avoir un père gai ou une mère lesbienne avec leurs amis et amies, aucun membre du groupe NA ne fait mention de cette ouverture.

Par contraste avec les NA, davantage de membres du groupe A mentionnent avoir eu des sentiments positifs en lien avec l'homosexualité du parent:

Je me rappelle, à une autre époque que j’ai sûrement aimée, il y avait la revue Treize [revue lesbienne québécoise]. Je sais que j'avais fait un article là dedans, j'avais dessiné quelque chose. J'étais bien contente qu'on me demande de parler de cela (fille de mère lesbienne $\mathrm{A}$ ).

Au primaire, j’aimais ma différence (fille de mère lesbienne A).

Je trouvais cela [son orientation gaie] juste cool, mon père était apprécié (garçon de père gai $A$ ).

À l'adolescence, davantage de membres du groupe NA rapportent avoir vécu des difficultés personnelles, telles que consommer de la drogue, avoir des problèmes d'identité, faire des attaques de panique, se chercher, ne pas étudier, aller dans une école spécialisée et abandonner l'école:

Moi, j’ai eu des problèmes de drogue. J'ai essayé un petit peu de tout. On dirait que j'essayais de me trouver, je me cherchais parce que j'étais super gêné (garçon de mère lesbienne NA). 


\section{LE RÔLE DE L’AFFIRMATION DE L’IDENTITÉ HOMOSEXUELLE}

Moi, je travaillais pas, je n’allais pas à l'école, je consommais [drogues], j'étais frustré (garçon de père gai NA).

D’autres membres du groupe NA mentionnent avoir entretenu des pensées homophobes:

C'est drôle, mais j'haïssais les homosexuels et j'emmagasinais tout ce que le monde disait, c'était ma plus grande peur d'être gai (garçon de mère lesbienne NA).

Toujours durant l'adolescence, plusieurs membres du groupe A rapportent avoir eu une bonne relation avec leur parent gai ou lesbien, contrairement à une majorité des NA qui rapportent une relation problématique avec ce parent:

Au même moment où j'avais encore des réticences avec ma mère, je me suis encore plus rapprochée d'elle. À partir de ce moment-là, la relation avec ma mère s'est améliorée (fille de mère lesbienne $\mathrm{A}$ ).

J'essayais toujours d'aller chercher l'amour de mon père. Même s’il était gai, je me demande pourquoi il ne m'acceptait pas et ne voulait pas me voir plus souvent que cela (garçon de père gai NA).

On remarque aussi que davantage de membres du groupe NA mentionnent être certains de l'orientation homosexuelle de leur parent malgré les efforts des parents d'afficher une identité hétérosexuelle. Un participant mentionne lors de l'entrevue plusieurs événements où son père fréquentait un homme qu'il nommait son colocataire:

Mon père ne me disait pas encore qu’il était gai. À l'adolescence, je me suis dis: « OK, c’est clair, je dois vivre avec ça! Qu'est-ce tu veux que je te dise! Il est gai! Il est gai! » tabou:

Aussi, pour plus de membres du groupe NA, l'homosexualité est encore un sujet

On est entré et on a vu mon père avec un gars, la vapeur qui sortait de la douche. Ils ont tellement changé de face quand on est entré dans la maison, venez pas me dire que c’est un ami, pourquoi ils étaient surpris comme ça? Il [son père] m’a dit: « Pourquoi tu ne lui as pas fait la conversation? C’est un de mes amis! » (garçon de père gai NA).

Certains disent avoir vécu des difficultés reliées au contexte familial, comme avoir souffert du manque de modèle masculin et avoir consulté un pédopsychiatre. Voici un garçon avec un père gai NA lui-même devenu gai à l'âge adulte:

Pour moi aussi, ça a été long avant d’affirmer ma propre homosexualité à ma famille parce que je me suis dit qu'il y avait déjà mon père qui faisait souffrir la famille à cause de son homosexualité cachée. Moi, je ne le ferai pas. C’est sûr que j’associais ça [l’homosexualité] à quelque chose de souffrant.

Comparés aux A, davantage de membres du groupe NA soulignent qu'ils parlaient de leur père gai ou de leur mère lesbienne, mais qu'en même temps, ils étaient dérangés par les réactions des autres:

Mes amis disaient: "Ton père est parti toi! Il est avec un autre gars, c'est une tapette! ». Ça me faisait de la peine (garçon de père gai NA).

J'ai eu une amie. C’était sa mère à qui ça dérangeait. Un jour, la mère de mon amie dit à mon amie: «Je te l'avais dit qu'elle aussi était lesbienne! ». J’ai été catégorisée comme ma mère et cela m'avait déçue (fille de mère lesbienne NA).

D’autres membres du groupe NA soulignent finalement qu'ils avaient peur de la réaction des autres dans le milieu scolaire:

Durant les 3 années de secondaire, j’ai essayé de garder ça le plus secret possible pour éviter justement de me faire taper dessus. Il y avait vraiment des personnes extrêmement anti-gaies à l'école; c'était vraiment fou (fille de père gai NA). 


\section{REVUE CANADIENNE DE SANTÉ MENTALE COMMUNAUTAIRE}

Plus de membres du groupe A que NA mentionnent toutefois avoir pris la défense de leur parent auprès de leurs amis et amies, dans leur réseau social et à l'école:

À l'école, il y avait des amis qui avaient des problèmes à l'accepter, mais moi, je leur avais dit simplement: "C'est mon père, alors je vais l'aimer comme il est [...] c'est mon choix ! ». J'ai perdu des amis à cause de ça (garçon de père gai A).

D’autres de ce groupe ont connu des réactions positives de l'entourage. Aussi, davantage de membres du groupe A mentionnent aux personnes qui fréquentent leur école qu'ils ont un père gai ou une mère lesbienne:

Je pense que cela allait de mieux en mieux, dans le sens où, au secondaire, un ou deux amis le savait (fille de mère lesbienne A).

\section{Maintenant}

Au moment de l'entrevue, plusieurs participants et participantes trouvent normal le fait d'avoir un parent homosexuel. Plusieurs autres mentionnent que d'avoir eu et d'avoir actuellement un parent homosexuel apportent un ajout à leur bagage personnel:

Autant un père gai c'est dur, autant je pense qu'en grandissant, j'ai été plus ouverte d'esprit que bien des gens. Il y a des personnes racistes, homophobes et fermées d'esprit (fille de père gai $A$ ).

Cela a été toute une expérience de vie finalement [d'avoir une mère lesbienne]. Je pense que cela m'a fortifié dans l'ensemble (garçon de mère lesbienne NA).

Certain(e)s, dérangés par les réactions négatives de certaines personnes, exigent maintenant des amis et amies capables d'accepter le fait qu'ils ont un parent homosexuel.

À l'âge adulte, plusieurs participants et participantes sont d'opinion que l'homosexualité ne définit pas une personne et qu'il faut en parler. Certain(e)s ont fait des recommandations pour que la vie des enfants de parents homosexuels soit vécue de façon plus positive. En voici quelques exemples: il faut davantage de programmes de prévention de l'homophobie et de sensibilisation envers l'homosexualité dans les écoles; les parents gais ou lesbiens devraient développer un lien de confiance avec leurs enfants; il est préférable de ne pas dire qu'on a un parent homosexuel aux personnes qui risquent de ne pas comprendre; il faut combattre la peur de l'homosexualité. D’autres ont décidé de s'impliquer socialement dans les causes qui traitent de l'homosexualité, par exemple, participer aux marches de la fierté gaie, rassembler des gens qui ont également vécu leur jeunesse auprès de parents gais ou lesbiens, créer des sites Internet de soutien et d'échanges pour enfants de parents non hétérosexuels, devenir militant ou militante ou encore travailler dans des organismes qui aident les proches des personnes gaies ou lesbiennes.

Pour ce qui est des propos qui distinguent les deux groupes à l'âge adulte, seuls les membres du groupe NA mentionnent avoir encore des réactions personnelles négatives envers l'homosexualité de leur parent, penser que leur parent n'aurait jamais dû avoir d'enfants, ressentir de la honte, du malaise ou encore du dégoût envers sa propre sexualité à cause de son parent homosexuel.

Je devais et dois mentir sur moi [le fait que le participant est gai]. C'est pénible de vivre dans le mensonge (garçon de mère lesbienne NA).

Le groupe NA se distingue également du groupe A par le fait que plus de membres de ce groupe-là, surtout de sexe masculin, ont mentionné avoir une relation problématique avec leur père gai ou leur mère lesbienne. D'autres de ce groupe mentionnent avoir des difficultés personnelles, comme être timide, avoir peur d'être fou et être freiné sexuelle- 


\section{LE RÔLE DE L’AFFIRMATION DE L’IDENTITÉ HOMOSEXUELLE}

ment devant une femme (pour les participants de sexe masculin), et ne pas accepter totalement ou avoir eu de la difficulté à accepter leur propre orientation homosexuelle:

À partir du moment où je me suis accepté comme gai, j'ai accepté ma mère lesbienne. Cela a été plus difficile de m'accepter. Il a été plus difficile de le dire à ma mère que j'étais gai. Ça a vraiment été très dur (garçon de mère lesbienne NA).

Je me suis dit, je ne serais jamais comme mon père gai parce qu'il n’a pas été honnête et il nous a fait souffrir. Je ne serai jamais comme lui, donc j'ai comme refoulé ça ou j'ai refusé finalement d'accepter mon orientation sexuelle (garçon de père gai NA).

Pour d'autres, toujours du groupe NA, l'âge adulte est encore une période difficile due au fait d'être l'enfant d'un père gai ou d'une mère lesbienne:

Aujourd'hui, c'est encore la même chose, je cours encore après mon père pour essayer d'avoir son amour (garçon de père gai NA).

Aussi, certains parents du groupe NA, selon les participants et participantes, n'acceptent pas encore le fait d'être gai ou lesbien et d'autres soulignent que leur parent a des difficultés personnelles. Ainsi, un participant de ce groupe révèle que son père est triste et une participante dit que sa mère trouve cela souffrant d'être lesbienne:

Souvent elles [sa mère et la conjointe de celle-ci] m’ont dit: « Si tu as quelque chose à

faire dans la vie, ne fais pas la même chose que nous, c'est trop dur à vivre ».

Finalement, certains interviewés et interviewées du groupe NA soulignent que leur père gai s'est retiré du noyau familial et plusieurs autres se disent encore dérangés par la non-affirmation du parent de sa propre homosexualité. Comparés au groupe A, des membres du groupe NA rapportent que l'homosexualité est encore un sujet tabou dans le contexte familial et ce, même à l'âge adulte. Ils disent qu'ils maintiennent à leur tour le tabou au sujet de l'homosexualité de leur parent auprès de leurs amis et amies, copains et copines.

En revanche, le groupe A devenu adulte se distingue du groupe NA par un grand nombre de membres qui continuent de percevoir positivement leur père gai ou mère lesbienne et entretiennent une bonne relation avec lui ou elle:

Notre relation est vraiment mieux, le fait qu'elle soit lesbienne ou pas, cela ne change plus rien (fille de mère lesbienne $\mathrm{A}$ ).

Davantage de membres du groupe A rapportent aussi des réactions positives de leur entourage social à l'homosexualité de leur parent, ils se sentent à l'aise et vont prendre la défense de leur parents auprès de leurs amis et amies, copains et copines, qui manifestent de la fermeture.

\section{DISCUSSION}

L'objectif de cette étude était de donner une voix à des adultes qui ont vécu leur enfance et leur adolescence durant les années 80 et 90 auprès d'un père gai ou d'une mère lesbienne né(e) dans les années 50 ou 60. Plus précisément, elle a tenté de mettre en lumière, par l'entremise d'entrevues semi-structurées, le vécu de ces enfants en fonction du degré d'affirmation, par le parent, de son identité sexuelle.

Eu égard aux résultats qui caractérisent l'ensemble des participants et participantes, ils révèlent que les participants et participantes ont presque tous vécu la séparation ou le divorce de leurs parents biologiques, surtout durant l'enfance, ce qui est en soi un facteur de stress empiriquement bien démontré. La majorité des participants et participantes ont également fréquemment gardé tabou la possibilité ou le fait que leur parent était gai ou 


\section{REVUE CANADIENNE DE SANTÉ MENTALE COMMUNAUTAIRE}

lesbien selon les circonstances et les personnes qu'ils fréquentaient. O’Connell (1993) rapporte des résultats semblables dans son étude dans laquelle les enfants adolescents de mères lesbiennes discriminaient les personnes à qui ils pouvaient parler de l'orientation sexuelle du parent en toute sécurité de celles à qui ils ne pouvaient pas parler. Une grande majorité des participants et participantes de notre étude ont craint la réaction des autres à l'idée que l'homosexualité de leur parent soit connue. Si le contexte hétérosexiste dans lequel ces enfants ont évolué peut expliquer ces résultats, on peut se demander dans quelle mesure les enfants d'aujourd'hui vivent avec la même nécessité de discriminer les environnements sécuritaires et non sécuritaires. Ces données soulèvent aussi la question du développement de la perception de la stigmatisation chez les enfants, et des stratégies développées par les parents pour assister leur enfant à gérer leur intégration dans des environnements sociaux homophobes. A ce jour, aucune recherche n’a été développée sur ces questions.

De façon générale, on constate que malgré les réactions négatives et positives des participants et participantes et leurs difficultés personnelles possiblement reliées au fait d'avoir un père gai ou une mère lesbienne, plusieurs enfants, à l'âge adulte, acceptent et prennent la défense de ce parent et nombreux sont ceux qui ont mentionné s'être affirmés comme enfant de parent homosexuel et qui ont trouvé enrichissant le fait d'être l'enfant d'un père gai ou d'une mère lesbienne. L'étude de Nadaud (2002) montre d'ailleurs que les enfants d'un parent homosexuel sont plus flexibles et tolérants que ceux de la population générale. Être l'enfant d'un parent homosexuel qui s'affirme amène probablement des défis particuliers, mais apporte également des avantages singuliers, tel l'apprentissage au respect de la diversité (Harris \& Turner, 1985/1986).

Eu égard aux distinctions entre les enfants d'un parent qui accepte sa propre orientation homosexuelle et ceux d'un parent qui ne l'accepte pas, les résultats montrent davantage d'inconfort et de réactions négatives chez les enfants dont les parents cachent leur orientation à leurs enfants, comparés aux enfants ayant des parents affirmatifs. Davantage de tabou chez les parents est associé à davantage de conflit entre les adolescents et adolescentes et leurs parents, davantage de tabou dans leur propre réseau, davantage d'anxiété sur leur orientation sexuelle et de difficulté à accepter leur propre orientation homosexuelle, ainsi que davantage de peur de la réaction des autres à l'idée que leur marginalité soit mise au jour. Ces résultats suggèrent que l'inconfort même des parents gais et lesbiennes par rapport à leur homosexualité se transmet à la génération suivante, qu'elle soit d'orientation hétérosexuelle ou homosexuelle.

D’ailleurs, ces données sont cohérentes avec celles de Rand, Graham et Rawlings (1982) selon lesquelles le bien-être psychologique des mères lesbiennes est associé à leur degré d'ouverture quant à leur identité de lesbienne et ce, entre autres auprès de leurs enfants. Inversement, les participants et participantes qui ont un parent qui affirme sa propre orientation homosexuelle se sont démarqués des autres, quant à eux, par plus de réactions positives et des relations plus harmonieuses entre le participant ou la participante et son père gai ou sa mère lesbienne. D'ailleurs, ils sont plus nombreux à divulguer à leur entourage l'identité sexuelle de leur parent, malgré le climat stigmatisant qu'ont connu et connaissent encore les personnes d'orientation homosexuelle. L'affirmation du parent de sa propre orientation homosexuelle semble affecter la qualité de vie de leurs enfants et être un facteur de protection.

L'affirmation des parents de leur propre orientation sexuelle repose toutefois, en partie, sur les contraintes imposées par des environnements hétérocentrés. Dans la présente étude, nous n'avons pas interviewé les parents de ces enfants. Des entrevues parentales 


\section{LE RÔLE DE L’AFFIRMATION DE L’IDENTITÉ HOMOSEXUELLE}

jumelées à celles des enfants auraient permis de contextualiser plus finement les réactions des enfants. Par exemple, la nécessité de certains parents de cacher leur orientation pour des raisons de garde d'enfant aurait permis de prendre l'entière mesure des conséquences, sur l'enfant, de politiques familiales discriminantes à l'époque. De telles entrevues auraient peut-être aussi facilité l'émergence d'autres dimensions des liens parent-enfant susceptibles d'expliquer la variabilité des réactions des enfants. Par exemple, une relation parentenfant conflictuelle avant même le développement d'une identité homosexuelle du parent crée des conditions de vulnérabilité qui risquent d'exacerber les effets négatifs des environnements homophobes et du silence des parents sur le bien-être des enfants.

Nos résultats montrent que la moitié des participants masculins de notre étude ont développé une identité homosexuelle. Ce résultat va à l'encontre de ceux des recherches empiriques sur la question qui montrent que, toute proportion gardée, les enfants de parents homosexuels ne sont pas plus nombreux que les autres à développer une identité homosexuelle (Bailey, Bobrow, Wolfe, \& Mikach, 1995; Bertrand, 1984; Bozett, 1980, 1982, 1987, 1989). Notre méthode de recrutement ciblant entre autres les minorités sexuelles en âge d'avoir des enfants adultes peut expliquer cette sur-représentation de garçons gais. Il est possible que les individus homosexuels ayant un parent homosexuel aient répondu plus favorablement à notre invitation que les garçons hétérosexuels parce qu'ils étaient plus à l'aise de parler de ces réalités avec un intervieweur. En effet, les recherches empiriques montrent généralement que les hommes hétérosexuels sont moins tolérants que les femmes envers l'homosexualité, et que les deux sexes sont moins tolérants envers l'homosexualité masculine qu'envers l'homosexualité féminine (e.g., Kite \& Whitley, 2003; Pilkington \& d'Augelli, 1995). Ceci pourrait entraîner une plus grande probabilité de participer à notre étude chez les filles hétérosexuelles de parents homosexuels que chez les garçons hétérosexuels de parents homosexuels.

À propos des limites de la présente étude, il importe de souligner que cette recherche s'inscrit dans un cadre exploratoire et ne se destine pas à être causale. Aussi, certaines dimensions non verbales ont échappé à la transcription écrite (Kvale, 1996). Bien que les grilles de classification aient été construites à partir des verbatim des participants et participantes, la présente recherche ne prétend pas présenter l'univers complet et complexe du discours auquel se réfèrent les énoncés.

Par ailleurs, la catégorisation des propos selon le degré d'affirmation par le parent de son orientation homosexuelle comporte aussi des limites puisqu'elle repose uniquement sur les représentations de leurs enfants. Des entrevues avec les parents de ces enfants auraient permis d'analyser le système des représentations des parents de manière à rendre compte de la complexité des situations familiales tout en fournissant des informations contextuelles sur les difficultés d'affirmation des parents. De plus, outre l'utilisation du nombre de propos reliés à l'affirmation du parent de sa propre orientation homosexuelle servant à catégoriser les participants et participantes selon les groupes A et NA, il serait intéressant d'analyser les répétitions de mots, de phrases ou d'expressions selon d'autres hypothèses que celle de l'affirmation. Dans un échantillon plus large, il serait important de comparer les garçons et les filles en intersection avec l'affirmation du parent puisque les deux sexes peuvent réagir différemment à l'homosexualité du parent (Bailey et al., 1995).

Les résultats de la présente étude peuvent s'avérer un guide pour des recherches cliniques ultérieures et suggèrent aux praticien(ne)s et professionnel(le)s de la santé de considérer l'impact de l'affirmation par le parent de sa propre orientation sexuelle sur le vécu de ses enfants. 


\title{
REVUE CANADIENNE DE SANTÉ MENTALE COMMUNAUTAIRE
}

\begin{abstract}
This study deals with the experiences, as reported by the participants, of 19 young adults (11 women and 8 men) who during their childhood and adolescence lived with a gay father or lesbian mother born in the 1950s or 1960s. The content of the interviews was classified and analyzed on the basis of categories inspired by Bronfenbrenner's (1986) ecological model and the effect of the parents' coming out in structuring their children's experience. Content analysis demonstrates that a child whose gay father or lesbian mother hides or does not accept his or her homosexual identity lives with greater discomfort and has more negative responses than a child whose parent affirms his or her homosexual identity. Results also show that a child with a parent who affirms his gay or her lesbian identity has more positive responses and relations with this parent than a child whose parent hides or does not accept his or her identity. Finally, the analysis indicates that children whose parents hide or do not accept their sexual identity have special difficulties in the transition from childhood to adolescence, such as fear of other people's reactions, internalized homophobia, and fear of becoming homosexual. These difficulties appear to persist into adulthood.
\end{abstract}

\section{RÉFÉRENCES}

Allen, M., \& Burrell, N. (1996). Comparing the impact of homosexual and heterosexual parents on children: Meta-analysis of existing research. Journal of Homosexuality, 32(2), 19-35.

Bailey, J.M., Bobrow, D., Wolfe, M., \& Mikach, S. (1995). Sexual orientation of adult sons of gay fathers. Developmental Psychology, 31, 124-129.

Barret, R.L., \& Robinson, B.E. (1990). Gay fathers. Lexington, MA: Lexington Books.

Bertrand, L. (1984). Le rapport Bertrand sur le vécu de 1000 femmes lesbiennes. Montréal: Les Éditions Primeurs.

Bigner, J.J., \& Bozett, F.W. (1989). Parenting by gay fathers. Marriage \& Family Review, 14, 155175.

Bigner, J.J., \& Jacobsen, R.B. (1989). Parenting behaviors of homosexual and heterosexual fathers. Journal of Homosexuality, 18, 173-86.

Bozett, F.W. (1980). How and why they disclose their homosexuality to their children. Family Relations, 29, 173-179.

Bozett, F.W. (1981). Gay fathers: Evolution of the gay-father identity. American Journal of Orthopsychiatry, 51, 552-559.

Bozett, F.W. (1982). Heterogeneous couples in heterosexual marriages: Gay men and straight women. Journal of Marital and Family Therapy, 8, 81-89.

Bozett, F.W. (1987). Children of gay fathers. Dans F.W. Bozett (dir.), Gay and lesbian parents (pp. 39-57). New York: Praeger.

Bozett, F.W. (1989). Gay fathers: A review of the literature. Dans F.W. Bozett (dir.), Homosexuality and the family (pp. 137-162). New York: Harrington Park Press.

Bronfenbrenner, U. (1986). Ecology of the family as a context for human development: Research perspectives. Developmental Psychology, 22(6), 723-742.

Deleury, E. (1984). Union homosexuelle et le droit de la famille. Les cahiers de droit, 25, 751-775.

Dunne, E.J. (1987). Helping gay fathers come out to their children. Journal of Homosexuality, 14, 213-222.

Editors of Harvard Law Review. (1990). Sexual orientation and the law. Cambridge, MA: Harvard University Press.

Green, R. (1992). Sexual science and the law. Cambridge, MA: Harvard University Press.

Harris, M.B., \& Turner, P.H. (1985/1986). Gay and lesbian parents. Journal of Homosexuality, 12, 101-113.

Herek, G. (1995). Psychological heterosexism in the United States. Dans Guidelines for psychotherapy with lesbian, gay, and bisexual clients (p. 5). Washington, DC: American Psychological Association. 


\section{LE RÔLE DE L’AFFIRMATION DE L’IDENTITÉ HOMOSEXUELLE}

Julien, D., Dubé, M., \& Gagnon, I. (1994). Le développement des enfants de parents homosexuels comparé au développement des enfants de parents hétérosexuels. Revue québécoise de psychologie, 15, 135-153.

Kite, M.E., \& Whitley, B.E., Jr. (2003). Do heterosexual women and men differ in their attitudes toward homosexuality?: A conceptual and methodological analysis. Dans L.D. Garnets \& D.C. Kimmel (dir.), Psychological perspectives on lesbian, gay, and bisexual experiences (2nd ed., pp. 165-187). New York: Columbia University Press.

Kvale, S. (1996). Interviews: An introduction to qualitative research interviewing. Thousand Oaks, CA: Sage.

Lapierre-Adamcyk, E. (2001). Évolution récente du mariage dans la société canadienne: les aspects démographiques et leur signification. Rapport de recherche soumis à Justice Canada.

Nadaud, S. (2002). Homoparentalité: une nouvelle chance pour la famille. Paris: Fayard.

O'Connell, A. (1993). Voices from the heart: The developmental impact of a mother's lesbianism on her adolescent children. Smith College Studies in Social Work, 63, 281-99.

Patterson, C.J. (2000). Family relationships of lesbians and gay men. Journal of Marriage and the Family, 62, 1052-1069.

Perez, R.M., DeBord, K.A., \& Bieschke, K.J. (dir.). (2000). Handbook of counselling and psychotherapy with lesbian, gay, and bisexual clients. Washington, DC: American Psychological Association.

Pilkington, N.W., \& d'Augelli, A.R. (1995). Victimization of lesbian, gay, and bisexual youth in community settings. Journal of Community Psychology, 23, 34-56.

Rand, C., Graham, D.L.R., \& Rawlings, E.I. (1982). Psychological health and factors the court seeks to control in lesbian mother custody trials. Journal of Homosexuality, 8(1), 27-39.

Savin-Williams, R.C., \& Esterberg, K.G. (2000). Lesbian, gay, and bisexual families. Dans D.H. Demo, K.R. Allen, \& M.A. Fine (dir.), Handbook of family diversity (pp.197-215). New York: Oxford University Press. 